\section{(1)}

CrossMark

\title{
No added value of interferon- $\gamma$ release to a prediction model for childhood tuberculosis
}

\author{
Toyin 0. Togun, Uzochukwu Egere", Marie P. Gomez', Abdou K. Sillah', \\ Mohammed Daramy ${ }^{1}$, Leopold D. Tientcheu ${ }^{1}$, Jayne S. Sutherland ${ }^{1}$, \\ Philip C. Hill ${ }^{2}$ and Beate Kampmann ${ }^{1,3}$
}

\begin{abstract}
Affiliations: 'Vaccines and Immunity Theme, Medical Research Council (MRC) Unit, Banjul, The Gambia. ${ }^{2}$ Centre for International Health and the Otago International Health Research Network, Dept of Preventive and Social Medicine, University of Otago School of Medicine, Dunedin, New Zealand. ${ }^{3}$ Academic Dept of Paediatrics, Imperial College London, London, UK.
\end{abstract}

Correspondence: Toyin 0. Togun, Vaccines and Immunity Theme, Medical Research Council (MRC) Unit, Atlantic Road, Fajara, P.0. Box 273 Banjul, The Gambia. E-mail: ttogunamrc.gm

ABSTRACT The predictive value of a combination of clinical and radiological features with interferon- $\gamma$ release assay (IGRA) for diagnosis of active tuberculosis (TB) disease among TB-exposed children is unknown.

150 symptomatic HIV-negative children (aged 3 months to 14 years), prospectively recruited through active contact tracing, were included. Backward stepwise logistic regression and bootstrapping techniques were used for the development and internal validation of a clinical prediction model for active TB disease. Model discrimination and incremental value of a positive IGRA test were assessed by area under the receiver operating characteristic curve (AUC).

$35(23 \%)$ children were diagnosed with active TB disease and started on treatment and $115(77 \%)$ had other respiratory tract infections. A final parsimonious clinical model, comprising age $<5$ years (adjusted (a)OR 4.8, 95\% CI 2.0-11.5) and lymphadenopathy on clinical examination (aOR 4.9, 95\% CI 1.8-13.0) discriminated active TB disease from other disease with an AUC of 0.70 (95\% CI 0.61-0.80). A positive IGRA result did not improve the discriminatory ability of the clinical model (c-statistic 0.72 versus 0.70 ; $\mathrm{p}=0.644$ ).

A clinical algorithm, including age $<5$ years and lymphadenopathy classified $70 \%$ of active TB disease among symptomatic TB-exposed children. IGRA does not add any discriminatory value to this prediction model.

@ERSpublications

No added value of interferon- $\gamma$ release to a prediction model for childhood TB in TB-exposed children http://ow.ly/S61CS

This article has supplementary material available from erj.ersjournals.com

Received: June 052015 | Accepted after revision: July 282015 | First published online: Oct 222015

Support statement: This work was supported by UK the Medical Research Council (MRC) and the UK Department for International Development (DFID) under the MRC/DFID concordat agreement (Childhood TB Research Programme grant reference number MR/K011944/1 to B. Kampmann) and the World Health Organization STOP TB/TBReach Wave 2 grant (reference number T9-370-114GAM). The funders had no involvement in the study design, collection, analysis and interpretation of the data nor in writing and submission of the report. Funding information for this article has been deposited with FundRef.

Conflict of interest: None declared.

Copyright CERS 2016 


\section{Introduction}

Tuberculosis (TB) is increasingly recognised as a leading cause of morbidity and mortality in children, and it is estimated that $75 \%$ of all childhood TB cases occur in 22 high-burden countries $[1,2]$. Children are usually exposed to Mycobacterium tuberculosis by an adult with infectious TB, with a high risk of developing active TB disease themselves, including extrapulmonary dissemination and death. This risk is particularly high in those aged $<2$ years unless screening and prophylactic treatment are implemented $[3,4]$. Evaluation of children with suspected intrathoracic TB is complicated by the poor specificity of the classical symptoms of TB and the low likelihood of microbiological confirmation due to the paucibacillary nature of childhood TB $[5,6]$. It has been suggested that clinical features may offer good prognostic value for active TB disease in children if they are well defined and appropriate risk stratification is applied [7].

A documented history of TB exposure in a child changes the pre-test probability of disease and the positive predictive value of subsequent investigations [5], providing an appropriate parameter for risk stratification. While a history of recent household exposure to an adult TB case has been shown to provide more diagnostic value than a positive tuberculin skin test (TST), particularly in HIV-infected children [7], data are sparse on the utility of interferon (IFN)- $\gamma$ release assays (IGRAs) in child contacts. IGRAs are known to be at least as sensitive but more specific than TST for detecting sensitisation to M. tuberculosis [8]. Neither TST nor IGRAs have been demonstrated to be superior in the diagnosis of latent TB infection in children nor for the prediction of active TB disease; importantly, neither can differentiate M. tuberculosis infection from active TB disease $[5,9,10]$. However, as has been suggested in previous reports, the use of IGRAs with other predictors might modify its predictive accuracy and offer utility in combination $[9,11,12]$. Therefore, we aimed to develop an optimal multivariable prediction model for active TB disease among symptomatic TB-exposed children with suspected intrathoracic TB disease using standard clinical, radiological and demographic variables, and investigate whether the use of IGRA as an adjunct test would add any further discriminatory value.

\section{Materials and methods}

\section{Setting and recruitment procedure}

A comprehensive childhood TB research programme was set up at the Medical Research Council (MRC) unit in the Gambia aiming at integrating clinical and epidemiological data with host and pathogen signatures. As part of this programme, active tracing of children (aged $<15$ years) who were living in the same compound with consecutively diagnosed adult smear-positive index TB cases was conducted between February 2012 and July 2014 in the Greater Banjul area of the Gambia where an estimated 80\% of all TB cases in the country are notified. A compound in the Gambia is a cluster of households often owned by members of the same family, as previously described $[13,14]$. In 2013, the incidence rate of TB in the Gambia was 173 cases/100000 population, while the prevalence of HIV among adults aged 15-49 years was $1.3 \%[15,16]$. Ethical approval for the study was obtained from the Gambia Government/MRC joint ethics committee.

Following written informed consent obtained from the parents/guardians of the children, household screening of the TB-exposed children was performed to assess them for possible TB disease using a standardised symptom screening questionnaire. In the clinical screening algorithm, symptoms suggestive of TB were defined as persistent, unremitting cough ( $\geqslant 14$ days) and/or fever and at least one of weight loss reported by the child's caregiver, reduced playfulness/activity, haemoptysis and night sweats. All children with symptoms suggestive of TB during the contact screening at recruitment were referred to the MRC childhood TB clinic for detailed clinical evaluation by study paediatricians, which included symptom review, physical examination and calculation of body mass index-for-age z-score and height-for-age z-score using the World Health Organization (WHO) 2007 reference standards [17]. All children referred to the clinic underwent HIV testing and a chest radiograph which was read independently by two study paediatricians, with any discordant report assessed by a third senior paediatrician who was blinded to the clinical data. A maximum of $5 \mathrm{~mL}$ of blood was drawn from each child by venepuncture for whole blood assay (WBA) and other host response studies. Sputum induction was requested for all children aged $\geqslant 3$ months with symptoms suggestive of $\mathrm{TB}$, with the respective samples sent for sputum microscopy, MTB/RIF assay (Xpert; Cepheid, Sunnyvale, CA, USA) and mycobacterial culture at the TB diagnostic laboratory on site. A TST was performed with a positive TST defined as transverse skin induration $\geqslant 10 \mathrm{~mm}$, regardless of bacille Calmette-Guérin vaccination status, measured within $48-72 \mathrm{~h}$ of intradermal injection of $0.1 \mathrm{~mL}$ of 2 tuberculin units of purified protein derivative (PPD RT23; Staten Serum Institute, Copenhagen, Denmark) in the volar aspect of the left forearm.

"Active TB disease" was defined according to the case definitions proposed by the WHO comprising both bacteriologically confirmed and clinically diagnosed TB cases [18], as described in table 1. All subjects diagnosed with active TB disease were referred for standard 6 months' TB treatment according to the 
TABLE 1 Diagnostic classification of tuberculosis (TB) according to revised World Health Organization case definitions

Confirmed TB

Clinically diagnosed TB ${ }^{\#}$

Detection of acid-fast bacilli by microscopy of secretions or; Identification of M. tuberculosis by culture or; Identification of M. tuberculosis by Xpert ${ }^{\text {I }}$

Does not fulfil criteria for bacteriological confirmation, but Suggestive appearance on chest radiograph and

Favourable response to specific antituberculous therapy $+/-$ positive tuberculin skin test

+/- suggestive histological appearances on biopsy material

M. tuberculosis: Mycobacterium tuberculosis. ${ }^{\#}$ : cases had suggestive symptoms and signs of TB, did not fulfil the criteria for bacteriological confirmation of disease, had suggestive appearance on chest radiograph and failed to respond to empirical broad-spectrum antibiotics. Favourable response to antituberculous therapy was an integral part of the clinical TB diagnosis. ": Xpert MTB/RIF assay (Cepheid, Sunnyvale, CA, USA).

paediatric TB treatment guidelines in the Gambia [19]. Children without bacteriological confirmation or radiological signs of active $\mathrm{TB}$ disease and with resolution of symptoms either spontaneously or with conventional short-course antibiotic treatment were diagnosed and treated for other respiratory infections but not TB ("other diseases"). All child contacts were followed up at home by trained field workers at 3-monthly intervals for 12 months, with repeated symptom screening and clinic visits if the child became unwell. Children diagnosed with active TB disease underwent clinic follow-up at 2 months and at the end of TB treatment with evaluation of their response to treatment.

Whole blood assay

A WBA was set up at the baseline evaluation within $4 \mathrm{~h}$ of venepuncture. $100 \mu \mathrm{L}$ of undiluted heparinised whole blood was incubated in duplicates with $M$. tuberculosis antigens ESAT-6/CFP-10 fusion protein (EC) $\left(10 \mu \mathrm{g} \cdot \mathrm{mL}^{-1}\right.$ final concentration; kindly provided by Tom Ottenhoff, Leiden University Medical Center, Leiden, the Netherlands) and positive (PHA-L; Sigma-Aldrich, Gillingham, UK; $10 \mu \mathrm{g} \cdot \mathrm{mL}^{-1}$ final concentration) and negative (RPMI 1640 medium; BioWhittaker, Verviers, Belgium) controls. After overnight incubation at $37^{\circ} \mathrm{C}$ with $5 \%$ carbon dioxide, supernatants were harvested, duplicates pooled and stored at $-20^{\circ} \mathrm{C}$ prior to analysis.

\section{Study participants}

Children were classified into three diagnosis groups following complete clinical assessment and investigation, as described earlier. These were: 1) bacteriologically confirmed TB; 2) clinically diagnosed TB; and 3) other disease. Children were included in this study and their respective WBA supernatants were selected for the in-house IGRA, according to the following criteria: 1) all children consecutively diagnosed with active TB disease and started on TB treatment between February 2012 and July 2014; and 2) a randomly selected sample of children diagnosed with other disease over the same period at a numerical ratio of 3:1 with all active TB disease cases.

\section{In-house IFN- $\gamma$ release assay}

The IFN- $\gamma$ concentration $\left(\mathrm{pg} \cdot \mathrm{mL}^{-1}\right)$ in the WBA supernatants of the active TB disease cases and other disease cases was determined by in-house IGRA in the TB immunology laboratory of the MRC unit in the Gambia as previously described [20, 21]. In brief, 96-well plates were coated with mouse anti-human IFN $-\gamma$ monoclonal antibody (final concentration $2 \mu \mathrm{g} \cdot \mathrm{mL}^{-1}$; Pharmingen, Oxford, UK) and incubated overnight at $4^{\circ} \mathrm{C}$. Following washing with PBS-5\% Tween-20, wells were blocked using PBS/fetal calf serum (Sigma-Aldrich, St Louis, MO, USA) for $2 \mathrm{~h}$. Samples, controls and standards were then added in duplicate and the plates incubated overnight at $4^{\circ} \mathrm{C}$. After washing on day 3, biotinylated mouse anti-human IFN- $\gamma$ detection antibody (final concentration $1 \mu \mathrm{g} \cdot \mathrm{mL}^{-1}$; Pharmingen) was added, the plates incubated for $45 \mathrm{~min}$ at room temperature and a final colorimetric step performed by addition of avidinperoxidase followed by OPD Fast (both from Sigma-Aldrich, USA). The reaction was stopped using $2 \mathrm{M}$ $\mathrm{H}_{2} \mathrm{SO}_{4}$ and the plates were read within $45 \mathrm{~min}$ at $492 \mathrm{~nm}$ with a four-parameter curve-fit. The background production of IFN- $\gamma$ measured in the negative control wells (medium only) was subtracted from that measured in the EC-stimulated wells to derive the M. tuberculosis antigen-specific IFN- $\gamma$ values. We defined a positive IFN- $\gamma$ response in the study subjects as $M$. tuberculosis antigen-specific IFN- $\gamma$ values $\geqslant 155.0 \mathrm{pg} \cdot \mathrm{mL}^{-1}$. This cut-off value was derived by calculating the mean plus two standard deviations of the IFN- $\gamma$ values measured in the unstimulated negative control wells, as previously described [20, 22]. 


\section{Statistical analysis}

All data used for statistical analysis in this study were derived from the clinical evaluation of the study participants at the MRC childhood TB clinic at the time of baseline contact investigation, and the in-house IGRA using WBA supernatants from the baseline evaluation of the respective children. The binary outcome variable for study subjects was defined as all active TB disease (comprising both bacteriologically confirmed and clinically diagnosed TB cases) compared to children with other disease. Basic demographic and clinical characteristics were compared between the outcome groups using nonparametric Wilcoxon rank-sum test for continuous variables and Chi-squared test for categorical variables. Table 2 outlines the definitions of the 14 candidate predictors known to be associated with childhood $\mathrm{TB}$, which were selected a priori based on clinical judgement and published literature [23, 24].

As previously suggested by Moons et al. [25], a reduced, most parsimonious multivariable clinical prediction model was derived by backward stepwise logistic regression, which started with all the candidate predictors except the IGRA test, with significance level for a variable to remain in the model set at $\mathrm{p}<0.1$. The selected clinical prediction model was extended with the addition of the IGRA test to assess the incremental value of a positive IFN- $\gamma$ response for predicting active TB disease. Internal validation of the backward stepwise predictor selection and of the final parsimonious clinical prediction model was performed using a bootstrapping technique with 1000 repetitions. Full details of the model selection process are provided in the online supplementary material. Calibration of the models was assessed by HosmerLemeshow goodness-of-fit test. The ability of the multivariable prediction models to discriminate between active TB disease and other disease was assessed by calculating the area under the receiver operating characteristic curve (AUC). All statistical analyses were performed using Stata software (version 13; StataCorp, College Station, TX, USA) and any $\mathrm{p}<0.05$ (two-sided) was considered statistically significant.

\section{Results}

Out of 3298 child contacts who received symptom screening in 952 households, 487 prospectively recruited symptomatic TB-exposed children with suspected intrathoracic TB underwent complete clinical investigations with final diagnostic classification over the recruitment period. Out of these 487 children, all 35 children diagnosed with active TB disease (comprising 16 bacteriologically confirmed TB and 19 clinically diagnosed TB cases) and a randomly selected sample of children with other disease $(n=115)$ at a numerical ratio of 3:1 with all active TB disease cases were included in the analysis (fig. 1). The 35 active TB disease cases were diagnosed consecutively at the time of contact screening and investigation. Therefore, a total of 150 symptomatic TB-exposed children with suspected intrathoracic TB disease were included in this analysis with a median (interquartile range) age of 6 (3-9) years; 76 (51\%) were male and none were HIV-infected (table 3). When stratified by the final diagnosis, children with active TB disease were younger and more likely to have lymphadenopathy on clinical examination compared to the children with other disease. However, we

\section{TABLE 2 Definitions of selected candidate predictors used in the study}

\section{Age years \\ Sex}

Cough: persistent and unremitting cough of $\geqslant 14$ days' duration

Weight loss: reported loss of weight by carer and/or objective evidence from child's growth chart with documented crossing of percentile line in the preceding 3 months

Fever: report of persistent fever ( $>1$ week) by carer and/or temperature $\geqslant 38^{\circ} \mathrm{C}$ at clinic evaluation Night sweats: drenching night sweat as indicated by soaked bedsheets or the clothing of the child Fatigue/malaise: persistent, unexplained reduced playfulness in an otherwise active child Lymphadenopathy: palpably enlarged cervical and/or other peripheral lymph nodes $\geqslant 2 \mathrm{~cm}$ in diameter (except inguinal lymph nodes)

Abnormal chest radiograph: independent report of abnormality that is consistent with active tuberculosis disease by two study physicians, with any discordant report assessed by a third senior clinician blinded to the clinic data of the index child

BCG vaccination: BCG scar seen on the left deltoid region and/or documented evidence of vaccination in child's immunisation card.

BMI-for-age z-score: BMI adjusted for age and sex using the WHO 2007 reference standard

Height-for-age z-score: height adjusted for age and sex the using WHO 2007 reference standard

Positive TST: skin test induration $\geqslant 10 \mathrm{~mm}$

Positive IFN- $\gamma$ response: Mycobacterium tuberculosis antigen-specific IFN- $\gamma$ values $\geqslant 155.0 \mathrm{pg} \cdot \mathrm{mL}^{-1}$ 


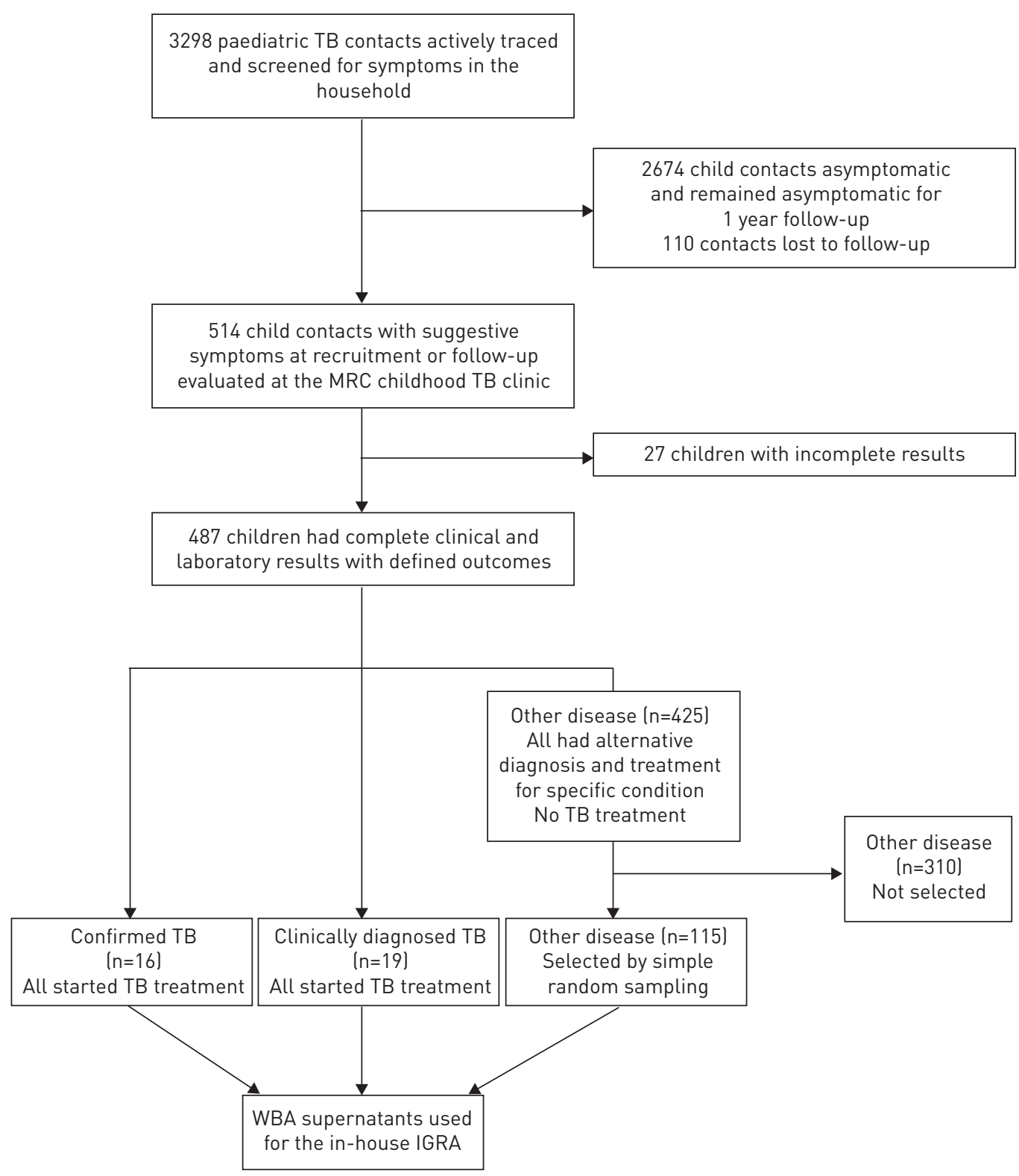

FIGURE 1 Recruitment and diagnostic classification of study subjects between February 2012 and July 2014. TB: tuberculosis; MRC: Medical Research Council; WBA: whole blood analysis; IGRA: interferon- $\gamma$ release assay.

found no significant difference between children with active TB disease compared to those with other disease with regard to the prevalence of each of the classical symptoms of TB (i.e. cough lasting $>2$ weeks, weight loss, fever and night sweats) and in the proportions with positive TST and IGRA results (table 3).

Development of a risk prediction model for active TB

In the univariable logistic regression analysis, age $<5$ years and presence of lymphadenopathy on clinical examination were found to be significant predictors of active TB disease (table 4). Although children with fever and fatigue/malaise were approximately twice as likely to have active TB disease when compared to those without these symptoms, the differences were of borderline statistical significance.

The clinical prediction multivariable regression model showed that age $<5$ years (adjusted (a)OR 4.8, 95\% CI 2.0-11.5) and presence of lymphadenopathy on clinical examination (aOR 4.9, 95\% CI 1.8-13.0) were strong, significant and independent predictors of active TB disease (table 5). The model showed good discriminatory ability for active TB disease with an AUC of 0.70 (95\% CI 0.61-0.80). The AUC of the multivariable clinical model was significantly higher than the AUC of each individual candidate predictor in the univariable analysis, which ranged between 0.52 and 0.60 (AUC comparison $\mathrm{p}<0.05$ in all). The model was then extended by the addition of a positive IGRA result, as shown in table 5 . Age $<5$ years and presence 
TABLE 3 Characteristics of tuberculosis (TB)-exposed children at baseline evaluation stratified by diagnosis of either active TB disease or other disease

\begin{tabular}{|c|c|c|c|c|}
\hline & All subjects & Active TB & Other disease & p-value \\
\hline Subjects & 150 & 35 & 115 & \\
\hline Age years & $6(3-9)$ & $4(2-7)$ & $7(4-9)$ & 0.004 \\
\hline$<5$ years & 55 (37) & $21(60)$ & $34(30)$ & 0.001 \\
\hline Sex male & $76(51)$ & $15(43)$ & $61(53)$ & 0.291 \\
\hline \multicolumn{5}{|l|}{ Clinical features } \\
\hline \multicolumn{5}{|l|}{ Symptoms } \\
\hline Cough lasting $\geqslant 2$ weeks & 105 (70) & $27(77)$ & $78(68)$ & 0.292 \\
\hline Weight loss & 80 (53) & $21(60)$ & $59(51)$ & 0.367 \\
\hline Night sweats & $51(34)$ & $16(46)$ & $35(30)$ & 0.095 \\
\hline Fever & $63(42)$ & $19(54)$ & $44(38)$ & 0.093 \\
\hline Fatigue/malaise & $32(21)$ & $11(31)$ & $21(18)$ & 0.096 \\
\hline \multicolumn{5}{|l|}{ Signs } \\
\hline Lymphadenopathy & $28(19)$ & $12(34)$ & $16(14)$ & 0.007 \\
\hline Abnormal chest radiography & $107(71)$ & $28(80)$ & 79 (69) & 0.195 \\
\hline$B C G$ vaccination & $99 / 141(70)$ & $21 / 33(64)$ & $78 / 108(72)$ & 0.345 \\
\hline BMI-for-age $z$-score $<-2$ SD & $44(29)$ & 13 (37) & $31(27)$ & 0.259 \\
\hline Height-for-age $z$-score $<-2$ SD & $20(13)$ & $4(11)$ & $16(14)$ & 0.705 \\
\hline \multicolumn{5}{|l|}{ Tests of TB infection } \\
\hline $\mathrm{TST} \geqslant 10 \mathrm{~mm}$ & 82 (55) & $17(49)$ & $65(57)$ & 0.408 \\
\hline IFN- $\gamma$ value $\geqslant 155.0 \mathrm{pg} \cdot \mathrm{mL}^{-1}$ & 103 (69) & $25(71)$ & 78 (69) & 0.687 \\
\hline \multicolumn{5}{|c|}{$\begin{array}{l}\text { Data are presented as } \mathrm{n} \text {, median (interquartile range), } \mathrm{n}(\%) \text { or } \mathrm{n} / \mathrm{N}(\%) \text {, unless otherwise stated. BCG: bacille } \\
\text { Calmette-Guérin; BMI: body mass index; TST: tuberculin skin test; IFN: interferon. }{ }^{\#} \text { : active TB disease } \\
\text { versus other disease. }\end{array}$} \\
\hline
\end{tabular}

of lymphadenopathy on clinical examination remained the significant, independent and strong predictors of active TB disease with adjusted odds ratios of 5.0 (95\% CI 2.1-11.9) and 4.8 (95\% CI 1.8-13.0) respectively, but there was no difference in the AUC between the IGRA-extended model and the clinical model (c-statistic 0.72 versus $0.70 ; \mathrm{p}=0.644$ ). Internal validation using a bootstrapping technique with 1000 repetitions gave normal-based $95 \%$ confidence intervals of the adjusted odds ratios for age <5 years (95\% CI 1.8-12.7) and lymphadenopathy (95\% CI 1.8-13.2), as well as the normal-based 95\% confidence interval of the AUC (95\% CI 0.61-0.79, bias 0.014; bootstrap standard error 0.048) that were similar to those of the non-bootstrapped

\section{TABLE 4 Univariable predictors of active tuberculosis (TB)}

OR $(95 \% \mathrm{CI})$

\begin{tabular}{lc}
\hline Demographics & \\
Age $<5$ years & $3.6(1.6-7.8)^{*}$ \\
Male & $0.7(0.3-1.4)$ \\
Symptoms & \\
Cough lasting $\geqslant 2$ weeks & $1.6(0.7-3.9)$ \\
Weight loss & $1.4(0.7-3.1)$ \\
Night sweats & $1.3(0.7-3.1)^{\#}$ \\
Fever & $1.9(0.9-3.7)^{\#}$ \\
Fatigue/malaise & $2.1(0.9-4.8)^{\#}$ \\
Signs & \\
Lymphadenopathy & $3.2(1.3-7.7)^{*}$ \\
Abnormal chest radiograph & $1.8(0.7-4.6)$ \\
BCG vaccination & $0.7(0.3-1.5)$ \\
BMI-for-age z-score <-2 SD & $1.6(0.7-3.6)$ \\
Height-for-age z-score <-2 SD & $0.8(0.3-2.6)$ \\
Tests of TB infection & \\
TST $\geqslant 10$ mm & $0.7(0.3-1.6)$ \\
Positive IFN- $\gamma$ response & $1.2(0.5-2.7)$
\end{tabular}

BCG: bacille Calmette-Guérin; BMI: body mass index; TST: tuberculin skin test; IFN: interferon. *: $p<0.05$; ${ }^{\#}: \mathrm{p}<0.1$. 
TABLE 5 Multivariable logistic regression models for predicting active tuberculosis (TB) disease

\begin{tabular}{|c|c|c|c|c|}
\hline & \multicolumn{2}{|c|}{ Model 1: clinical prediction } & \multicolumn{2}{|c|}{$\begin{array}{l}\text { Model 2: clinical prediction } \\
\text { with IFN- } \gamma \text { response }\end{array}$} \\
\hline & Coefficient & aOR & Coefficient & aOR \\
\hline \multicolumn{5}{|l|}{ Clinical } \\
\hline Intercept & $-2.25 \pm 0.37$ & & $-2.47 \pm 0.51$ & \\
\hline Age $<5$ years & $1.58 \pm 0.44$ & $4.8(2.0-11.5)^{*}$ & $1.60 \pm 0.45$ & $5.0(2.1-11.9)^{*}$ \\
\hline Positive IFN- $\gamma$ response & & & $0.29 \pm 0.48$ & $1.3(0.5-3.3)$ \\
\hline \multicolumn{5}{|l|}{ Incremental value performance measures } \\
\hline Hosmer-Lemeshow goodness-of-fit $p$-value & & \multicolumn{2}{|c|}{0.525} \\
\hline AUC $(95 \% \mathrm{CI})$ & \multirow{2}{*}{\multicolumn{2}{|c|}{$0.70(0.61-0.80)$}} & \multicolumn{2}{|c|}{$0.72(0.62-0.82)$} \\
\hline AUC comparison $p$-value ${ }^{\Uparrow}$ & & & \multicolumn{2}{|c|}{0.644} \\
\hline Bayesian information criterion $^{+}$ & \multicolumn{2}{|c|}{158} & \multicolumn{2}{|c|}{$\begin{array}{c}162 \\
0.524\end{array}$} \\
\hline
\end{tabular}

Data are presented as coefficient \pm SE or adjusted (a)OR $(95 \% \mathrm{CI})$, unless otherwise stated. IFN: interferon; AUC: area under the receiver operating characteristic curve. "\#: a test of model calibration with a null hypothesis that there is no difference between observed and model predicted probabilities in which a p-value closer to 1 suggest good calibration; " ${ }^{\text {I: }}$ comparing the clinical model with the IFN- $\gamma$ extended model; ${ }^{+}$: a measure of efficiency used for comparing the predictive accuracy of maximum likelihood models, which combines model fit and complexity; a smaller value suggests lower prediction error; ${ }^{\S}$ : a test for the clinical model nested within the IFN- $\gamma$ extended model with $p<0.05$, indicating that the added predictor is statistically important to the model. *: $p<0.05$.

clinical prediction model. The final clinical model, comprising the combination of age $<5$ years and lymphadenopathy on clinical examination, selected six out of the 35 children diagnosed with active TB disease $(17.2 \%, 95 \%$ CI $6.6-33.7 \%)$ and only one of 115 children with other disease, giving a very high specificity of $99.1 \%$ (95\% CI $95.3-100 \%)$ with positive and negative predictive values of $85.7 \%$ (95\% CI $42.1-99.6 \%)$ and $79.7 \%(72.2-85.9 \%)$, respectively.

In further restricted analysis shown in table 6 , age $<5$ years (aOR 3.2, 95\% CI 1.1-9.9) and lymphadenopathy on clinical examination (aOR 3.9, 95\% CI 1.1-14.1) were also significant and independent predictors of bacteriologically confirmed TB, and the clinical model discriminated confirmed TB with an AUC of 0.67

TABLE 6 Multivariable logistic regression models for predicting bacteriologically confirmed tuberculosis (TB) disease

\begin{tabular}{|c|c|c|c|c|}
\hline & \multicolumn{2}{|c|}{ Model 1: clinical prediction } & \multicolumn{2}{|c|}{$\begin{array}{l}\text { Model 2: clinical prediction } \\
\text { with IFN- } \gamma \text { response }\end{array}$} \\
\hline & Coefficient & aOR & Coefficient & aOR \\
\hline \multicolumn{5}{|l|}{ Clinical } \\
\hline Intercept & $-2.73 \pm 0.47$ & & $-3.87 \pm 0.87$ & \\
\hline Age $<5$ years & $1.15 \pm 0.58$ & $3.2(1.1-9.9)^{*}$ & $1.28 \pm 0.60$ & $3.6(1.1-11.6)^{*}$ \\
\hline Positive IFN- $\gamma$ response & & & $1.37 \pm 0.80$ & $3.9(0.8-18.9)$ \\
\hline \multicolumn{5}{|l|}{ Incremental value performance measures } \\
\hline Hosmer-Lemeshow goodness-of-fit p-value & & 0.631 & & 0.161 \\
\hline $\operatorname{AUC}(95 \% \mathrm{Cl})$ & & $0.67(0.51-0.79)$ & & $0.73(0.62-0.83)$ \\
\hline AUC comparison $p$-value ${ }^{\pi}$ & & & & 0.061 \\
\hline Bayesian information criterion ${ }^{+}$ & & 105 & & 106 \\
\hline
\end{tabular}


(95\% CI 0.51-0.79). Similarly, extension of the clinical model with the addition of IGRA did not improve the discriminatory ability of the clinical model for bacteriologically confirmed TB (c-statistic 0.73 versus 0.67 ; $\mathrm{p}=0.061)$.

\section{Discussion}

Differentiating active TB disease from other respiratory infections is one of the most pressing challenges in the management of children presenting with symptoms and signs that are not specific for TB. In this study, we found that symptomatic TB-exposed children aged $<5$ years or those with lymphadenopathy on clinical examination were at least four times as likely to have active TB disease compared to their respective comparison groups. In addition, a positive IFN- $\gamma$ response did not add any further discriminatory value to the simple clinical algorithm of age $<5$ years and lymphadenopathy on clinical examination, which could reliably distinguish $\geqslant 70 \%$ of active $\mathrm{TB}$ disease cases from other disease in this study population. While microbiological confirmation of TB disease in children is an exception rather than the rule, similarly we found that symptomatic TB-exposed children aged $<5$ years or with lymphadenopathy on clinical examination were at least thrice as likely to have bacteriologically confirmed TB relative to their respective comparison groups.

The candidate predictors of active TB disease in this study were selected based on judgement of their relevance to the clinical presentation of childhood $\mathrm{TB}$, ease of ascertainment and from published reports relevant to the epidemiology of childhood TB in resource-limited settings [23, 26]. As expected, we found no difference between children with active TB disease and other disease in our study in the prevalence of chest radiographs with abnormality reported as consistent with active TB disease, given the poor specificity of chest radiography in childhood TB. Similarly, in a further restricted analysis, we found no difference between children with only bacteriologically confirmed TB compared with other disease in the prevalence of such abnormal chest radiograph reports, and it was neither a significant predictor of confirmed TB nor all active TB disease. In addition, radiography facilities are unavailable in many resource-limited settings, or available only in secondary or tertiary health facilities where cost is restrictive and reporting is subject to wide inter- and intra-observer variability [5]. Our finding that the classical symptoms of TB, including persistent unremitting cough lasting $\geqslant 2$ weeks, fever, weight loss and night sweats, were not significant predictors of active TB disease, further confirms the poor specificity of these symptoms in children, as reported in earlier studies [23, 24].

Studies from both high- and low-TB prevalence areas have shown that young children aged $<5$ years can mount robust IFN- $\gamma$ responses to $M$. tuberculosis antigens, comparable to those of older children [27, 28]. In this study, we found that a positive IGRA result is not a predictor of active TB disease, in keeping with earlier reports, which is attributable to the inability of IGRAs to distinguish between active TB disease and M. tuberculosis sensitisation $[9,29]$. However, we also found that a positive IFN- $\gamma$ response did not add any discriminatory value to the ability of a multivariable clinical prediction model to discriminate between active TB disease and other disease. Equally, IFN- $\gamma$ release added no value to the performance of the clinical model when the analysis was restricted to only children with bacteriologically confirmed TB or other disease, with the clinical model reliably distinguishing $\geqslant 67 \%$ of bacteriologically confirmed $\mathrm{TB}$ disease cases from other disease in this study population. This in itself is unique and interesting given the paucity of data on IGRA in children in TB-endemic settings in general, but particularly in relation to the predictive value of a positive IGRA results for active TB, especially when used as an adjunct test in multivariable clinical algorithms. Studies have suggested that IFN- $\gamma$ might be necessary, but not sufficient on its own, for prediction of active TB disease, especially in countries with a high burden of $\mathrm{TB}$, and that a combination of other risk factors with IGRA results might have much higher predictive ability than IFN $-\gamma$ response alone $[9,11]$. However, our result that assessed IGRA results in a multivariable risk prediction model for prevalent active TB disease, applied in an exclusively paediatric active contact tracing study setting, did not support this assertion.

This study had some limitations. The study population were TB-exposed children in a TB-endemic setting with low HIV prevalence, identified by household contact-tracing, and the findings may therefore not be generalisable to all children suspected of having TB or to areas with high HIV/TB co-infection. Similarly, $\sim 70 \%$ of our study population had evidence of sensitisation to $M$. tuberculosis by IGRA, and thus the finding may not be generalisable to areas of low TB prevalence. As such it is essential to validate the findings in settings with different TB and HIV epidemiological contexts. In addition, we did not use the clinical case definitions proposed for classification of intrathoracic TB by an expert panel convened by the National Institute of Allergy and Infectious Diseases/National Institutes of Health because, as discussed in depth by the authors, the proposed case definitions may not be appropriate for studies that incorporate active investigation of possible TB in children from household case-finding studies [30].

In conclusion, we report that IGRA does not add any further incremental value to the discriminatory ability of a clinical prediction model that includes age $<5$ years and presence of lymphadenopathy on 
clinical examination, which reliably distinguished $\geqslant 70 \%$ of active $\mathrm{TB}$ disease from other disease among symptomatic TB-exposed children with suspected intrathoracic TB. Although this clinical algorithm has a low sensitivity for diagnosis of active TB disease in these children, it correctly classified children with other disease with a very high specificity of $99.1 \%$, and the model could select children with high or low risk of active TB disease with positive and negative predictive values of $\geqslant 80 \%$. As such, while the clinical algorithm should not be used as a diagnostic tool to make decisions whether to initiate TB treatment or not, it could be a very simple and efficient tool to identify symptomatic TB-exposed children with even higher risk of active TB in resource-limited settings for more intense follow-up investigations. This could potentially result in earlier diagnosis and initiation of TB treatment in general, but even more specifically important and useful in the context of the WHO-recommended provision of isoniazid prophylaxis for all TB contacts aged $<5$ years where there is the crucial need to rule out active TB disease first.

\section{Acknowledgements}

We acknowledge the Medial Research Council (MRC) childhood TB field and clinic teams (field workers and supervisors, field and nurse coordinators, etc.) who facilitated the recruitment, screening and follow-up of the study subjects. We also acknowledge Schadrac Christin Agbla for his statistical advice and Fatoumata Cole (both Department of Statistics and Data Management, MRC Unit, Banjul, The Gambia) for general data management support and the entire TB diagnostic laboratory staff. We thank the parents/guardians and families of the study subjects who participated in this research project.

\section{References}

1 Graham SM, Sismanidis C, Menzies HJ, et al. Importance of tuberculosis control to address child survival. Lancet 2014; 383: 1605-1607.

2 Nelson LJ, Wells CD. Global epidemiology of childhood tuberculosis. Int J Tuberc Lung Dis 2004; 8: 636-647.

3 Guwatudde D, Nakakeeto M, Jones-Lopez EC, et al. Tuberculosis in household contacts of infectious cases in Kampala, Uganda. Am J Epidemiol 2003; 158: 887-898.

4 Marais BJ, Gie RP, Schaaf HS, et al. The clinical epidemiology of childhood pulmonary tuberculosis: a critical review of literature from the pre-chemotherapy era. Int J Tuberc Lung Dis 2004; 8: 278-285.

Perez-Velez CM, Marais BJ. Tuberculosis in children. N Engl J Med 2012; 367: 348-361.

Newton SM, Brent AJ, Anderson S, et al. Paediatric tuberculosis. Lancet Infect Dis 2008; 8: 498-510.

Marais BJ, Gie RP, Hesseling AC, et al. A refined symptom-based approach to diagnose pulmonary tuberculosis in children. Pediatrics 2006; 118: e1350-e1359.

8 Pai M, Zwerling A, Menzies D. Systematic review: T-cell-based assays for the diagnosis of latent tuberculosis infection: an update. Ann Intern Med 2008; 149: 177-184.

9 Rangaka MX, Wilkinson KA, Glynn JR, et al. Predictive value of interferon- $\gamma$ release assays for incident active tuberculosis: a systematic review and meta-analysis. Lancet Infect Dis 2012; 12: 45-55.

10 Pollock L, Basu Roy R, Kampmann B. How to use: interferon $\gamma$ release assays for tuberculosis. Arch Dis Child Educ Pract Ed 2013; 98: 99-105.

11 Moons KG, Grobbee DE. Diagnostic studies as multivariable, prediction research. J Epidemiol Community Health 2002; 56: 337-338.

12 Moons KG, van Es GA, Michel BC, et al. Redundancy of single diagnostic test evaluation. Epidemiology 1999; 10: 276-281.

13 Adetifa IM, Ota MO, Jeffries DJ, et al. Commercial interferon gamma release assays compared to the tuberculin skin test for diagnosis of latent Mycobacterium tuberculosis infection in childhood contacts in the Gambia. Pediatr Infect Dis J 2010; 29: 439-443.

14 Hill PC, Brookes RH, Adetifa IM, et al. Comparison of enzyme-linked immunospot assay and tuberculin skin test in healthy children exposed to Mycobacterium tuberculosis. Pediatrics 2006; 117: 1542-1548.

15 World Health Organization. Global Tuberculosis Report 2014. http://apps.who.int/iris/bitstream/10665/137094/1/ 9789241564809_eng.pdf?ua=1 Date last accessed: March 1, 2015.

16 UNAIDS. Global Report: UNAIDS Report on the Global AIDS Epidemic 2013. www.unaids.org/sites/default/files/ media_asset/UNAIDS_Global_Report_2013_en_1.pdf Date last accessed: December 4, 2014.

17 World Health Organization. WHO Child Growth Standards: Methods and Development. Length/Height for Age, Weight for Age, Weight for Length, Weight for Height and Body Mass Index for Age. Geneva, World Health Organization, 2007.

18 World Health Organization. Definitions and Reporting Framework for Tuberculosis - 2013 Revision. www.who. int/iris/bitstream/10665/79199/1/9789241505345_eng.pdf Date last accessed: December 3, 2014. Date last updated: December 2014.

19 Gambian National Leprosy and Tuberculosis Control Programme. Gambian National Leprosy and Tuberculosis Control Programme: National Guidelines for the Management of Tuberculosis. Banjul, Department of State for Health, The Gambia, 2012.

20 Black GF, Thiel BA, Ota MO, et al. Immunogenicity of novel DosR regulon-encoded candidate antigens of Mycobacterium tuberculosis in three high-burden populations in Africa. Clin Vaccine Immunol 2009; 16: 1203-1212.

21 Sutherland JS, Lalor MK, Black GF, et al. Analysis of host responses to Mycobacterium tuberculosis antigens in a multi-site study of subjects with different TB and HIV infection states in sub-Saharan Africa. PLoS One 2013; 8: e74080.

22 Kassa D, Ran L, Geberemeskel W, et al. Analysis of immune responses against a wide range of Mycobacterium tuberculosis antigens in patients with active pulmonary tuberculosis. Clin Vaccine Immunol 2012; 19: 1907-1915.

23 Schaaf HS, Beyers N, Gie RP, et al. Respiratory tuberculosis in childhood: the diagnostic value of clinical features and special investigations. Pediatr Infect Dis J 1995; 14: 189-194.

24 Marais BJ, Gie RP, Obihara CC, et al. Well defined symptoms are of value in the diagnosis of childhood pulmonary tuberculosis. Arch Dis Child 2005; 90: 1162-1165. 
25 Moons KG, Kengne AP, Woodward M, et al. Risk prediction models: I. Development, internal validation, and assessing the incremental value of a new (bio)marker. Heart 2012; 98: 683-690.

26 Marais BJ, Obihara CC, Gie RP, et al. The prevalence of symptoms associated with pulmonary tuberculosis in randomly selected children from a high burden community. Arch Dis Child 2005; 90: 1166-1170.

27 Lewinsohn DA, Zalwango S, Stein CM, et al. Whole blood interferon-gamma responses to Mycobacterium tuberculosis antigens in young household contacts of persons with tuberculosis in Uganda. PLoS One 2008; 3: e3407.

28 Critselis E, Amanatidou V, Syridou G, et al. The effect of age on whole blood interferon-gamma release assay response among children investigated for latent tuberculosis infection. J Pediatr 2012; 161: 632-638.

29 Machingaidze S, Wiysonge CS, Gonzalez-Angulo Y, et al. The utility of an interferon gamma release assay for diagnosis of latent tuberculosis infection and disease in children: a systematic review and meta-analysis. Pediatr Infect Dis J 2011; 30: 694-700.

30 Graham SM, Ahmed T, Amanullah F, et al. Evaluation of tuberculosis diagnostics in children: 1. Proposed clinical case definitions for classification of intrathoracic tuberculosis disease. Consensus from an expert panel. J Infect Dis 2012; 205: Suppl. 2, S199-S208. 